\title{
Editorials
}

\section{The genetics of osteoarthritis}

Osteoarthritis is the most frequent cause of musculoskeletal disability in developed countries. Although the multifactorial nature of osteoarthritis is well recognised, ${ }^{1}$ the role of genetic factors in the development of osteoarthritis has recently received considerable attention with the development of molecular biology techniques.

For over 50 years certain forms of osteoarthritis have been thought to have a strong genetic component. Early studies by Stecher in $1941^{2}$ demonstrated that Heberden's nodes of the fingers were three times as common in the sisters of 64 affected subjects as in the general population. Family studies in the early 1960 s of individuals with generalised osteoarthritis suggested that first degree relatives were twice as likely to have radiographic generalised disease too. ${ }^{3}$

Further suggestive evidence for a genetic predisposition to osteoarthritis has come from the association of osteoarthritis with some HLA haplotypes ${ }^{4}$ and $\alpha$-1-antitrypsin isoform patterns. ${ }^{4}$ However, not all studies supported this. ${ }^{5}$ There are also a number of rare subtypes of osteoarthritis, including familial calcium pyrophosphate deposition disease, Stickler syndrome and some chondrodysplasis, that have a genetic basis and are associated with severe, early onset osteoarthritis.

Recently, a clear genetic influence on osteoarthritis was demonstrated in a study of 500 unselected female twins aged 45-70 years who were screened radiologically for osteoarthritis of the hands and knees. ${ }^{6}$ The correlations of osteoarthritis disease status were consistently twofold higher in 130 pairs of identical compared with 120 non-identical twin pairs. The influence of genetic factors was estimated to be between 35 and $65 \%$, independent of known environmental or demographic confounders.

Genetic linkage analysis of large kindred populations has been performed to examine some potential candidate genes in osteoarthritis. Several unrelated families have demonstrated co-inheritance of primary generalised osteoarthritis with specific alleles of the gene for type II procollagen (COL2A1) on chromosome $12 .^{7}$ This allele has now been cloned and found to be normal except for a single base mutation at position 519 of the $\alpha 1$ (II) chain, ${ }^{8}$ which was found in all affected members of a family but in none of the unaffected or unrelated individuals. Linkage analysis of several Stickler syndrome kindreds has also demonstrated that this disease is linked to COL2A1 in about $25-50 \%$ of families.

Despite these exciting developments, there is currently little evidence that the common forms of osteoarthritis are due to collagen mutations. The families described earlier are extremely rare and associated with mild chondrodysplasias. A recent study used gene specific highly polymorphic markers and affected sibling pair analyses to investigate genetic linkage between generalised osteoarthritis and three cartilage matrix genes: COL2A1, which encodes type II collagen; CRTL1, which encodes the cartilage link protein; and CRTM, which encodes the cartilage matrix protein. ${ }^{10}$ No linkage between generalised osteoarthritis and these genes was shown, suggesting that these genes are not major susceptibility loci for generalised osteoarthritis. A recent study which analysed blood leucocyte DNA to detect mutations in the gene for COL2A1 showed that mutations could be detected in up to $2 \%$ of patients with early onset familial osteoarthritis. ${ }^{11}$ This suggests that only a small proportion of cases of osteoarthritis can be explained by this genetic abnormality. Recently, promising new data has suggested an association between polymorphisms of the vitamin $\mathrm{D}$ receptor, which has recently been associated with osteoporosis, and early knee osteoarthritis. ${ }^{12}$ As some evidence suggests an inverse association between these two common conditions, it may be that some genes may have a number of effects on bone and cartilage.

Primary generalised osteoarthritis is likely to be a heterogeneous disease at the genetic level and mutations in genes other than COL2A1 are likely to be responsible for this phenotype. Yet to be explored is the role of mutations in the genes encoding the minor collagen types (for example, IX, X, XI). For example, transgenic mouse models with a central deletion in the $\alpha 1$ chain of the type IX collagen gene suggest that this gene may be important as heterozygotes develop osteoarthritis with no signs of chondrodysplasia. ${ }^{13}$ Also, the role of the other extracellular matrix proteins such as aggrecan, decorin and the link protein will need to be investigated.

In summary, genetic factors have a major role in osteoarthritis. This genetic influence has now been estimated to lie between 39 and $65 \%$ based on a recent female twin study. The nature of the genetic influence in osteoarthritis is speculative and may involve either a structural defect (that is, collagen) or alterations in cartilage or bone metabolism. Exciting work has identified mutations in type II collagen to be important in some rare, familial forms of osteoarthritis but the genetic basis of the common forms of osteoarthritis is currently unknown. Finding the genes involved is likely to lead to important new therapeutic targets and diagnostic advances in this common, disabling disease.

FLAVIA M CICUTTINI

Senior Lecturer,

Department of Epidemiology and Preventive Medicine,

Monash University,

Australia

Consultant Rheumatologist/Honorary Senior Lecturer

TIM D SPECTOR

Department of Rheumatology,

St Thomas's Hospital, Guys' and St Thomas's Trust,

London SE1 7EH

1 Anderson JJ, Felson DT. Factors associated with osteoarthritis of the knee in the first national health and nutrition examination survey (NHANES I). Am 7 Epidemiol 1988;128:179-89.

2 Stecher RM. Heberden's nodes. Heredity in hypertrophic arthritis of the finger joints. $A m \mathcal{F} M e d$ S $i$ 1941;201:801

3 Kellgren JH, Lawrence JS, Bier F. Genetic factors in generalised osteoarthritis. Ann Rheum Dis 1963;22:237-55.

4 Pattrick M, Manhire A, Ward M, Doherty M. HLA-A, B antigens and $\alpha-1$ antitrypsin phenotypes in nodal and generalized osteoarthritis and erosive osteoarthritis. Ann Rheum Dis 1989;48:470-5.

5 Benavides G, Cerantes A, Silva B, Kantona G, Lardizabal J. HLA and Herbeden's nodes in Mexican mestizos. Clin Rheumatol 1985;4:97-8.

6 Spector TD, Cicuttini F, Baker J, Loughlin J, Hart DJ. Genetic influences on osteoarthritis: a twin study. $B M \mathcal{F} 1996 ; 312: 940-4$ 
7 Palotie A, Vaisanen P, Ott J, Ryhanen L, Elima K, Vikkula M, et al. Predisposition to familial osteoarthritis linked to type II collagen gene. Lance position to famili

8 Alla-Kokko L, Baldwin CT, Moskovitz RW, Prockop DJ. A single base mutation in the type II procollagen gene (COL2A1) as cause of primary osteoarthritis associated with a mild chondrodysplasia. Proc Natl Acad Sci USA 1990;87:6565-8.

9 Francomano CA, Liberfarb RM, Hirose T, Maumenee IH, Streeten EA, Meyers DA, et al. The Stickler syndrome: Evidence for the close linkage to the structural gene for type II collagen. Genomics 1987;1:293-6.

10 Loughlin J, Irven C, Fergusson C, Sykes B. Sibling pair analysis shows no linkage of generalized osteoarthritis to the loci encoding type II collagen, cartilage link protein or cartilage matrix protein. $B r f$ Rheumatol 1994;33:1103-6.

11 Ritvaniemi P, Korkko J, Bonaventure J, Vikkula M, Hyland J, Paassilta P, et al. Identification of COL $2 \mathrm{~A} 1$ gene mutations in patients with chondrodysplasias and familial osteoarthritis. Arthritis Rheum 1995;38:999-1004

12 Griffith GO, Keen RW, Hart DJ, Nandra D, Lanchbury JS, Doyle DV, et al. Polymorphisms of the vitamin D receptor and osteoarthritis [abstract]. $\mathrm{Br}$ f Rheumatol 1996;35:117.

13 Nakata K, Ono K, Miyazaki J, Olsen BR, Muragaki Y, Adachi E, et al. Osteoarthritis associated with mild chondrodysplasia in transgenic mice $T$ expressing $\alpha \mathrm{l}(\mathrm{IX})$ collagen chains with a central deletion. Proc Natl Acad Sci USA 1993;90:2870-4.

\section{The pathology of osteogenesis imperfecta}

Osteogenesis imperfecta is a group of related, inherited disorders of connective tissues affecting approximately 1 in 30000 of the population. ${ }^{1}$ The basic defect is abnormal synthesis of type I collagen molecules.

There are four main classes of osteogenesis imperfecta, designated I-IV with subclasses. Each class is named by reference to the genetic abnormality or clinical features, or both. Thus type I is known as dominant with blue sclerae; type II as lethal perinatal; type III as progressive deforming; and type IV as dominant with normal sclerae.

As the names indicate, some forms of the disease are transmitted as autosomal dominant disorders, and by inference, others have autosomal recessive characteristics. The four classes encompass numerous gene defects caused by deletions, mutations and insertions in the genes controlling formation of type I collagen. ${ }^{2}$

Collagen is synthesised from three promolecules known as alpha chains. Within any type of collagen the chains may be all the same or different, but structurally related, molecules. The chains have designations such as alpha 1 (I) (the alpha 1 chain type I collagen). In the cell each alpha chain is altered, by cleavage, hydroxylation and glycosylation, before being combined with the other two to form a triple helix. The resultant molecule is known as procollagen.

The procollagen molecule is exported from the cell and, in the extracellular environment, the triple helix region is cleaved from its propeptides and assembled into collagen microfibrils. These then polymerise, often with other types of collagen, to form fibrils.

The genetic defects in osteogenesis imperfecta affect intracellular events in the process of synthesis and assembly of type I procollagen. Specifically, each subclass is characterised by abnormalities in the genes encoding the synthesis of the pro-alpha 1 (I) or pro-alpha 2 (I) chains, resulting in formation of (a) insufficient normal molecules; (b) unstable molecule; or (c) molecules that will not form helices. A transgenic model exists in mice in which a mutant gene for pro-alpha 1 (I) has been introduced into the mouse genome. ${ }^{3}$

The most consistently described effect of abnormal type I collagen synthesis is a relative imbalance in the ratio of type I to type III collagen. Because of the way these two collagens are incorporated into the polymerised fibril, the most striking abnormality on ultrastructural examination of the tissue is a decrease in collagen fibre size. ${ }^{4}$

Pathologically, the effects of these abnormal collagen fibrils are seen in those tissues which are particularly reliant on collagen fibre integrity for normal function. These include bone matrix, sclerae, dentine, tendons, blood vessels, and skin. ${ }^{5}$ Osteogenesis imperfecta usually presents with the consequence of poor bone formation and $\$$

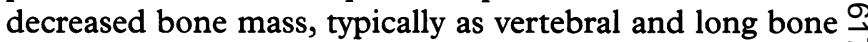
fracture. In addition, the sclerae may appear blue because 0 they are thinned and translucent, permitting partial visualisation of the underlying choroid; there are complex changes in tooth formation leading to small blue-yellow misshapen teeth; tendons and ligaments become lax, $\stackrel{\complement}{\infty}$ permitting an abnormal degree of joint movement; blood $\vec{\theta}$ vessel walls become weakened; and the texture and $\&$ resilience of skin becomes abnormal.

As its name suggests type II osteogenesis imperfecta has a very poor outlook. The altered collagen structure makes all the bones "brittle" and, as a consequence, the child is born with multiple fractures leading to trauma to the brain and poor ventilation.

Because it is a rare disease the pathological features of other forms of osteogenesis imperfecta tend to be reported anecdotally. In this issue of the Journal, McAllion and Paterson ${ }^{6}$ examine, for the first time, the causes of death in a sufficiently large series of patients with osteogenesis? imperfecta to relate them to disease type.

It is well recognised that patients with type I osteogenesis imperfecta have a normal stature, but have an element $\frac{0}{3}$ of skeletal fragility, abnormalities of dentition, hearing impairment (due to compression of the eighth nerve by bony abnormalities), lax joints, and blue sclerae; class IV $\frac{7}{0}$ patients have similar, but less noticeable disorders. McAllion and Paterson have shown that despite the disease these of patients have a normal life expectancy.

By contrast, the patients with type III osteogenesis imperfecta who, in life, have growth retardation, multiple 0 fractures, progressive kyphoscoliosis, hearing impairment, 0 and abnormal dentition, are at risk of dying prematurely from diseases secondary to their underlying collagen $\stackrel{?}{?}$ abnormality. This paper discusses the mechanism of death in these patients, but, in many ways it is what is not said $\underset{\mathbb{D}}{\stackrel{O}{d}}$ that is as important as what is. The specific structural abnormalities in the type I collagen molecule in each sub- 0 class are known. McAllion and Paterson, by highlighting the mode of dying, identify a method in which the 8 functional significance of specific parts of the collagen molecule can be related to the structural integrity of the tissue.

\section{A J FREEMONT}

Osteoarticular Pathology,

Department of Pathological Sciences,

Stopford Building,

University of Manchester,

Oxford Road,

Manchester M13 9PT 